\title{
THE RECENT PERFORMANCE OF THE OMEGA RICH DETECTOR IN EXPERIMENT WA89 AT CERN*)
}

\author{
U. Müller ${ }^{1,2)}$, W. Beusch ${ }^{2)}$, M. Boss ${ }^{3, a)}$, J. Engelfried ${ }^{3, b)}$, S. G. Gerassimov ${ }^{4, c)}$, \\ W. Klempt ${ }^{2}$, P. Lennert ${ }^{3)}$, K. Martens ${ }^{3)}$, D. Newbold ${ }^{5)}$, H. Rieseberg ${ }^{3)}$, \\ H.-W. Siebert ${ }^{3)}$, V.J. Smith ${ }^{5)}$, O. Thilmann ${ }^{3)}$ and G. Wälder ${ }^{3, d}$ )
}

\begin{abstract}
The hyperon beam experiment WA89 at CERN uses the upgraded Omega RICH detector for identification of $\pi, K$ and $p / \bar{p}$ from $\Sigma^{-}-N$ reactions. Cherenkov photons from a $5 \mathrm{~m}$ long nitrogen radiator are detected in drift chambers with TMAE-loaded ethane. Recent results on the performance of the detector are presented.
\end{abstract}

\author{
Presented at the \\ RICH95 International Workshop on RICH Detectors, \\ Uppsala, Sweden, 12-16 June 1995 \\ (To be published in Nucl. Instr. Meth. A)
}

*) Work supported by the Bundesministerium für Forschung und Technologie under contract numbers 05 5HD15I, 06 HD524I, and 06 MZ5265 TP2.

1) Institut für Kernphysik, Univ. Mainz, Germany.

2) Div. PPE, CERN, Geneva, Switzerland.

3) Physikalisches Institut, Univ. Heidelberg, Germany.

4) Max-Planck-Institut für Kernphysik, Heidelberg, Germany.

5) Department of Physics, Univ. Bristol, United Kingdom.

a) Now at SAP AG, Walldorf, Germany.

b) Now at Fermilab, Batavia, Ill., USA.

c) On leave from Lebedev Phys. Inst., Moscow, Russia.

d) Now at Charmilles Technologies SA, Meyrin, Switzerland. 



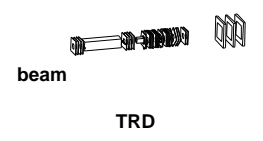

Target area

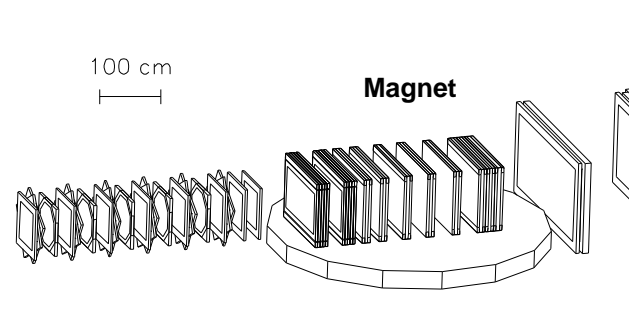

DC,MwPC
Decay area

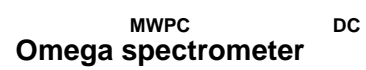

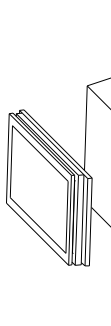

Hod 1

RICH

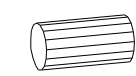

Lead SpaCal
Glass

Hod 2 Calorimeters

Figure 1: Experimental setup of WA89 in 1993 and 1994.

\section{$1 \quad$ Introduction}

The RICH detector at the Omega facility of the CERN SPS was - in its original design - first operated in 1984 for the photoproduction experiment WA69 [1] and later on for hadroproduction in WA82 [2]. In 1989, a major upgrade of the detector took place. It included a replacement of the central part of the mirror array and of the photosensitive drift chambers and their gas system. The detector was then used in several beam times of the hyperon beam experiment WA89, which will be described in detail below, and in the heavy-ion experiment WA94, as described in D. Elia's talk [3].

\section{The hyperon beam experiment WA89}

The main physics goals of WA89 are:

- The study of charmed-strange baryons, e.g. the decay $\Omega_{c}^{0} \rightarrow \Xi^{-} K^{-} \pi^{+} \pi^{+}$, and the measurement of their branching ratios and lifetimes [4].

- The search for exotic particles, e.g. $U(3100)^{+} \rightarrow \Lambda \bar{p} \pi^{+} \pi^{+}[5,6]$.

- The study of $\Xi$ and $\Omega$ resonances, and of polarization phenomena in hyperon production [7].

Most of the states studied include a charged kaon or an (anti-)proton as daughter particles. In the presence of a pion background at least ten times as large, particle identification is an important requirement for all these measurements. The mean momentum of the hyperon beam is $340 \mathrm{GeV} / \mathrm{c}$, leading to a momentum spectrum of secondaries extending up to about $100 \mathrm{GeV} / \mathrm{c}$. The necessary angular resolution for this momentum required a replacement of the central part of the mirror array of the existing Omega RICH by mirrors of higher optical quality and the construction of new photosensitive drift chambers with better resolution. The active surface of the photon detector is $160 \times 75 \mathrm{~cm}^{2}$, in order to detect Cherenkov light from particles with momenta down to $12 \mathrm{GeV} / \mathrm{c}$.

The apparatus of WA89 is designed as a forward spectrometer. It consists of a target area, a decay zone, and the Omega magnetic spectrometer, followed by the RICH detector and calorimeters [8]. A schematic view of this setup, as used during the 1993 and 1994 beam times, is shown in Fig. 1.

\section{$3 \quad$ The Omega RICH in WA89}

An overall view of the RICH detector, indicating the positions of the mirrors and the chambers, is shown in Fig. 2. Particles traversing the $5 \mathrm{~m}$ long radiator volume generate Cherenkov light which is focused by an array of hexagonal mirrors. The array consists of 19 mirrors of $44 \mathrm{~cm}$ diameter made from a glass / glass foam sandwich, surrounded by 74 mirrors of $70 \mathrm{~cm}$ diameter made from moulded glass panes. All mirrors are coated with 


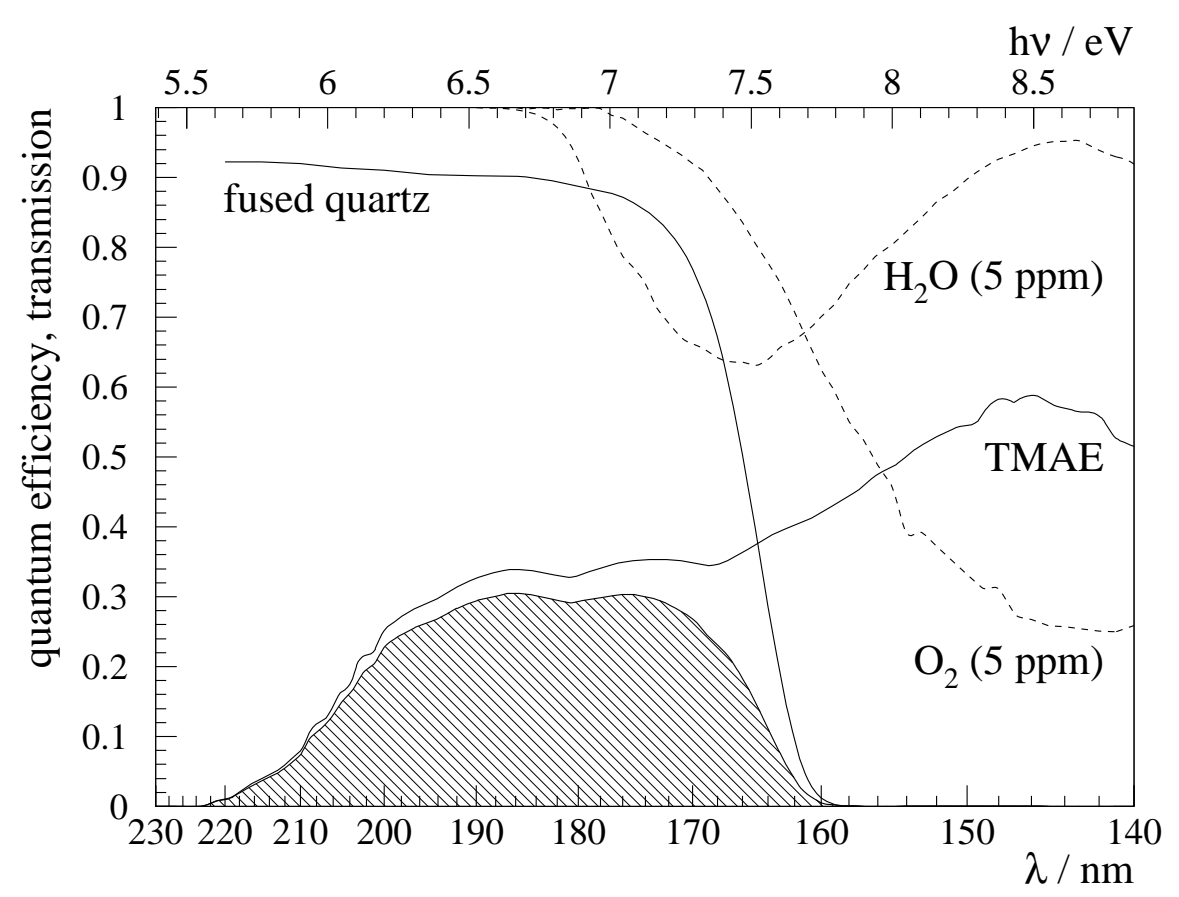

Figure 3: TMAE quantum efficiency and quartz transmission. The effects of 5 ppm water or oxygen contamination in the $5 \mathrm{~m}$ long radiator are also indicated.

surface absorb humidity from the air. It takes several weeks until they are outgassed again and the water vapour contamination of the radiator reaches the level of 3-4 ppm. For oxygen, a level of below 2 ppm was easily achieved.

During operation of the $\mathrm{RICH}$, the radiator gas is continuously cleaned by circulating it through oxygen and water absorbers containing activated copper and silica-gel, respectively. About two exchanges of the $120 \mathrm{~m}^{3}$ large volume are performed per day. The recirculation and pressure control system for the radiator is described in ref. [1]. The oxygen and water content of the radiator gas are monitored and its UV transmission spectrum is measured frequently, using a monochromator and a $4 \mathrm{~m}$ long absorption cell.

\subsection{The drift chambers}

Figure 4 shows one of the five drift chambers. It has two symmetrical parts separated by a common central high-voltage electrode. UV photons enter the chamber through the entrance window and create photoelectrons, which are guided by a homogeneous drift field towards the counting wires at the lower and upper end of the chamber, respectively.

The entrance windows are fused quartz panes with a size of $34.5 \times 81.0 \mathrm{~cm}^{2}$ and a thickness of $3 \mathrm{~mm}$. They carry conductive strips on both sides, with a width of $40 \pm 5 \mu \mathrm{m}$ and a pitch of $1.27 \mathrm{~mm}$. The transmission of the quartz sheets was measured in a vacuum test station. It showed a cut-off at $160 \mathrm{~nm}$ and reached $80 \%$ at $180 \mathrm{~nm}$ for windows with strips. The side and back walls of the drift volume are made of epoxy-fibreglass material ("FR4"). They carry conductive strips of $0.5 \mathrm{~mm}$ width, with $1.27 \mathrm{~mm}$ pitch, which are connected to the central electrode by a resistor chain.

The potential strips on the entrance window and the walls are arranged to provide a constant drift field pointing away from the window at an angle of $50 \mathrm{mrad}$, in order to avoid losing photoelectrons by diffusion onto the window. Because of this, the depth of the sensitive volume varies from $40 \mathrm{~mm}$ at the central electrode to $60 \mathrm{~mm}$ near the counting wires. 


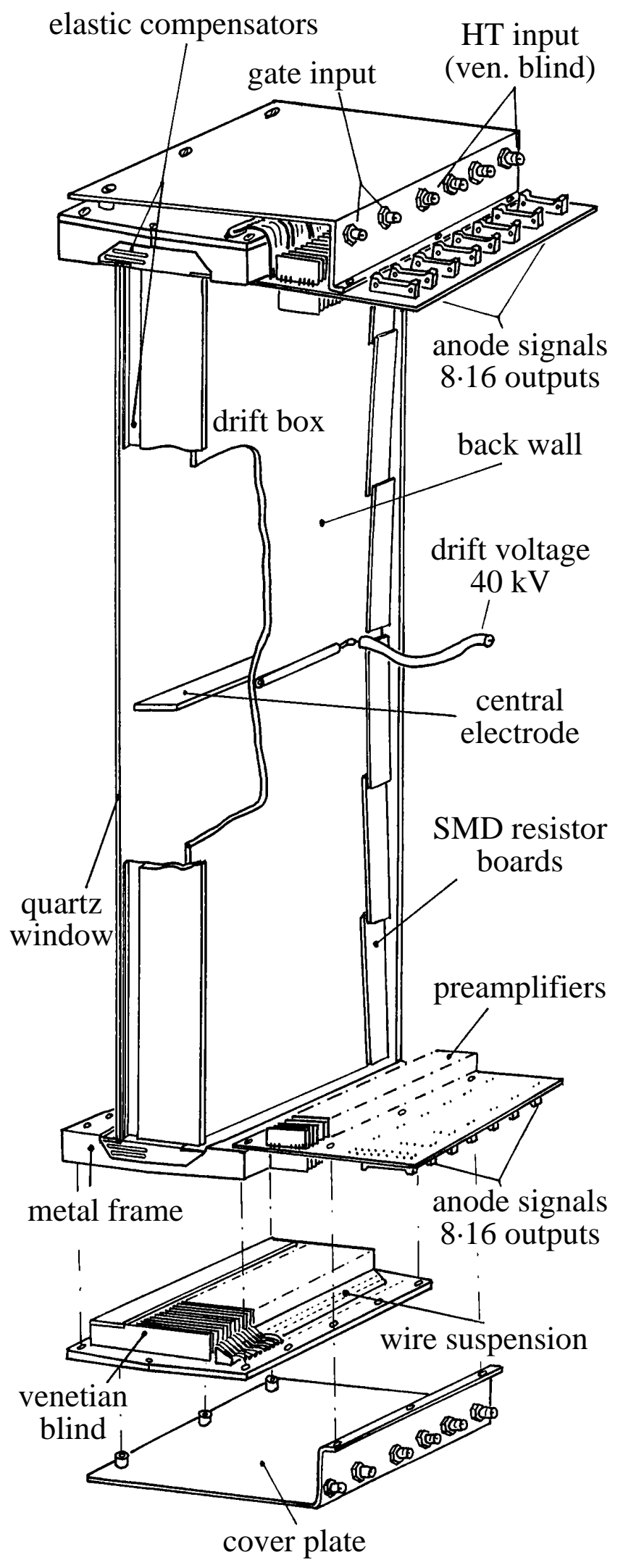

Figure 4: Design of one chamber, seen from the rear. The lower counting region is shown in an exploded view. 
Each chamber has two detachable counting modules, at its upper and lower end respectively. Each module contains 128 counting wires with $2.54 \mathrm{~mm}$ pitch and a diameter of $15 \mu \mathrm{m}$. For the definition of the individual counting cells and for suppression of photon crosstalk, separator plates made from $\mathrm{Al}_{2} \mathrm{O}_{3}$ ceramics are placed between the counting wires, forming a venetian blind structure. The plates carry conductive strips for field shaping and gating. More details on the chamber construction can be found in [11].

Ethane is used as counting gas, chosen for its good counting properties and low diffusion coefficients [12]. The drift field, generated by supplying a potential of $-40 \mathrm{kV}$ to the central electrode of the chamber, is $0.92 \mathrm{kV} / \mathrm{cm}$. This results in a drift velocity of $5.3 \mathrm{~cm} / \mu \mathrm{s}$.

\subsection{Gas and monitoring systems}

Great care was taken to ensure the purity of the counting gas. The ethane is cleaned by passing it through chromium trioxide absorbers ${ }^{1)}$, resulting in an oxygen content of less than $0.1 \mathrm{ppm}$. For the gas system, stainless steel tubing and metal seals were used wherever possible. Before operation, the whole system was evacuated and helium leak tested. To minimize oxygen contamination from leaks, a protective volume at the back side of the chambers is flushed with nitrogen. The residual oxygen content of the gas at the chamber exhaust was measured to be 1 ppm or less, in the absence of TMAE.

The TMAE was cleaned ${ }^{2)}$ by the vacuum distillation process developed for DELPHI [13]. The gas is saturated with TMAE vapour in a bubbler at a temperature of $30{ }^{\circ} \mathrm{C}$. This necessitates heating the RICH and the gas supply system to temperatures of $40^{\circ} \mathrm{C}$ or above to avoid any danger of TMAE condensation.

Temperatures at various points of the detector and the gas system, the pressures inside the chambers and the radiator, the gas flows into each chamber, the oxygen content of the ethane, high voltages, and currents are continuously measured and written to tape. In case of an exceeded limit, an alarm is generated to alert the shift crew. In addition to this software monitor, the oxygen content of the gas before the TMAE bubbler and several temperatures have hardware limits. If one of these is exceeded, or in case of a power failure, a system of pneumatic valves causes the ethane to bypass the TMAE bubbler.

\subsection{Gating}

Under typical experimental conditions, the chambers are passed by about $10^{6}$ charged particles in one SPS spill of $2 \mathrm{~s}$. Therefore, the counting action of the chambers must be gated $[11,14]$ in order to suppress field distortions from space charges and to reduce possible ageing effects. This is achieved by supplying static potentials to the uppermost strips of the venetian blinds such that the transfer of electrons to the counting wires is inhibited. After a trigger, the field configuration is "opened" by a high-voltage pulse for a time of $8 \mu \mathrm{s}$, corresponding to the maximum drift time. The total duty cycle of open/closed time is 0.5-1\%. For the 1993 and 1994 beam times, with all chambers operated in gated mode, no wire ageing effects have been observed.

\subsection{Readout}

The signals from the counting wires are amplified by current-sensitive preamplifiers mounted on the counting modules (Fig. 4). The amplifier outputs are connected to discriminators, placed outside the RICH box. In addition to the single electron thresholds

1) "Oxisorb", Messer Griesheim GmbH, Industriegase, D-40474 Düsseldorf.

2) Bayer AG, Abt. ZF-TVT 3, Thermische Trenntechnik, D-51368 Leverkusen. 


\begin{tabular}{|l|c|c|}
\hline error & $\sigma_{\theta, y} / \mathrm{mrad}$ & $\sigma_{\theta, z} / \mathrm{mrad}$ \\
\hline multiple scattering & \multicolumn{2}{|c|}{$4.3 \mathrm{GeV} / \mathrm{pc}$} \\
refractive index & \multicolumn{2}{|c|}{0.02} \\
chromatic dispersion & \multicolumn{2}{|c|}{0.35} \\
mirror alignment & \multicolumn{2}{|c|}{0.1} \\
astigmatism & $1.1 \cdot 10^{-5} \mathrm{~cm}^{-2} \cdot \mathrm{b}^{2}$ \\
parallax error & $4.1 \cdot 10^{-3} \mathrm{~cm}^{-1} \cdot \mathrm{b}$ \\
\hline track angle & 0.07 & 0.40 \\
electron diffusion & 0.23 & 0.15 \\
digitizing error & 0.15 & 0.05 \\
\hline total & 0.49 & 0.59 \\
\hline
\end{tabular}

Table 1: Expected contributions to the single photon angular resolution, in horizontal $(y)$ and vertical $(z)$ direction. The total error is calculated for a pion with a momentum $p=40 \mathrm{GeV} / \mathrm{c}$ and an impact parameter $b=30 \mathrm{~cm}$ to the mirrors' centre of curvature.

provided individually for each counting wire, a second higher threshold can be set for groups of eight neighbouring wires, in order to tag signals from charged particle tracks. The signals are then digitized by multi-hit TDCs.

\section{Detector performance}

\subsection{Angular resolution}

The expected contributions to the angular resolution for single Cherenkov photons are listed in Table 1. The diffusion coefficients for ethane were taken from ref. [12]. It should be noted that the errors from several photons of the same ring are correlated. For the contributions from track angle measurement and multiple scattering, the single photon errors tend to cancel each other when the Cherenkov ring radius is calculated.

The experimental resolution is determined by measuring the distance of every hit to the ring centres predicted from tracks observed in the spectrometer. Fig. 5 shows a distribution of these distances vs the particle momentum, taken from a 1993 sample of standard interaction triggers. By fitting a Gauß distribution to the pion signal for a small momentum band $(50-55 \mathrm{GeV} / \mathrm{c})$, a width $\sigma_{r}=0.28 \mathrm{~cm}$ can be determined, corresponding to a single photon angular resolution $\sigma_{\theta}=0.58 \mathrm{mrad}$.

\subsection{Photoelectron statistics}

During the beamtime, the number of photoelectrons per ring is estimated from rings of beam particles. For about $70 \%$ of all beam triggers, one non-interacting and nondecaying beam particle traverses the radiator. In a display of timings vs wire addresses (Fig. 6) a clear "beam ring" can be seen. If one looks at an appropriate group of wires, their timing distribution shows a narrow peak from which the number $N_{\text {obs }}$ of photoelectrons per beam particle is obtained. The advantage of this procedure compared to offline analysis is that the number $N_{\text {obs }}$ can be measured about once per hour. Thus, possible problems can be discovered quickly and tuning of parameters during detector setup is possible. Furthermore, no alignment and calibration are needed for this method. Fig. 7 shows $N_{\text {obs }}$ vs the run number in the 1994 beam time, for a total time of 80 days. The slope at the beginning corresponds to the removal of water vapour from the radiator. 


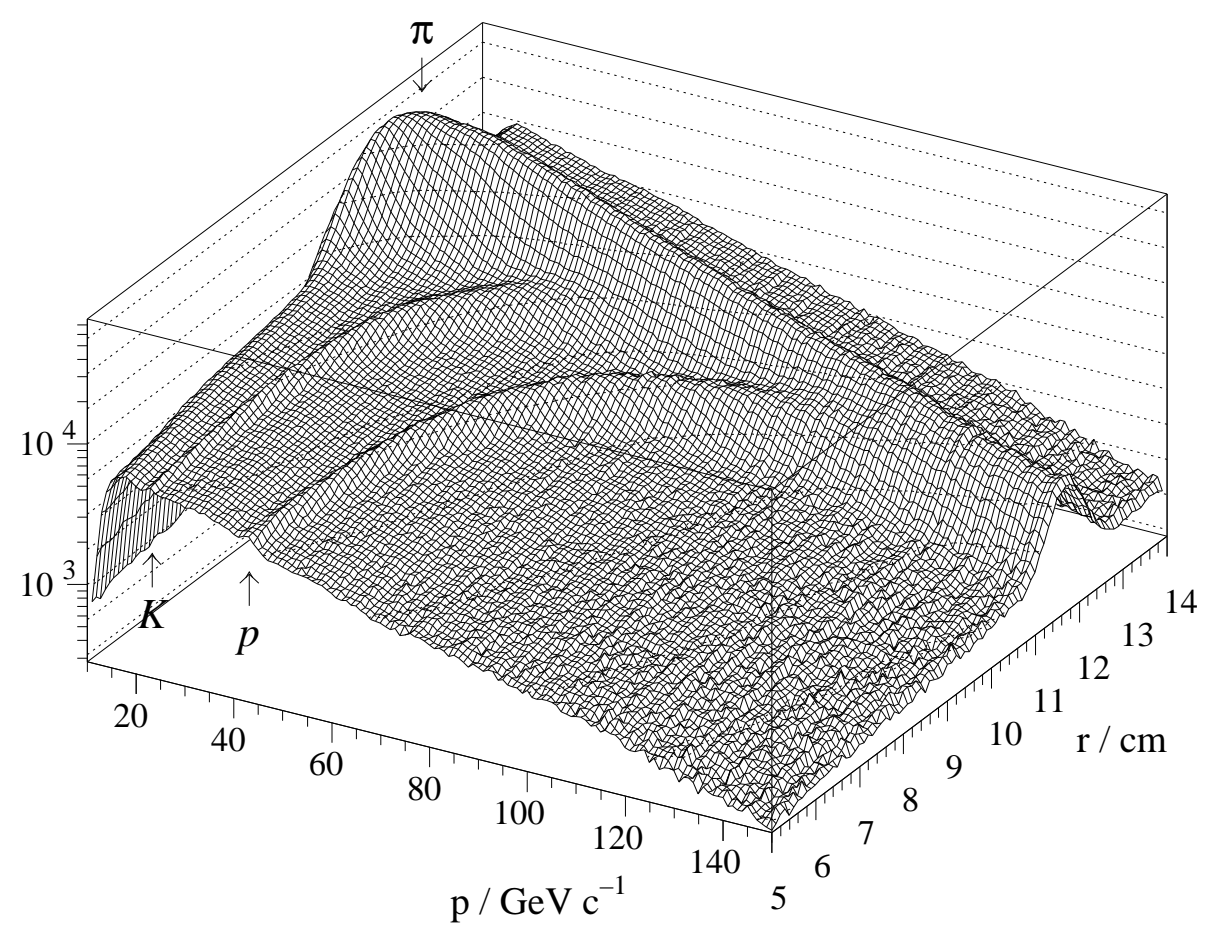

Figure 5: Distance $r$ of single hits to the predicted ring centres vs particle momentum $p$. The signals for $\pi, K$, and $p / \bar{p}$ are indicated.

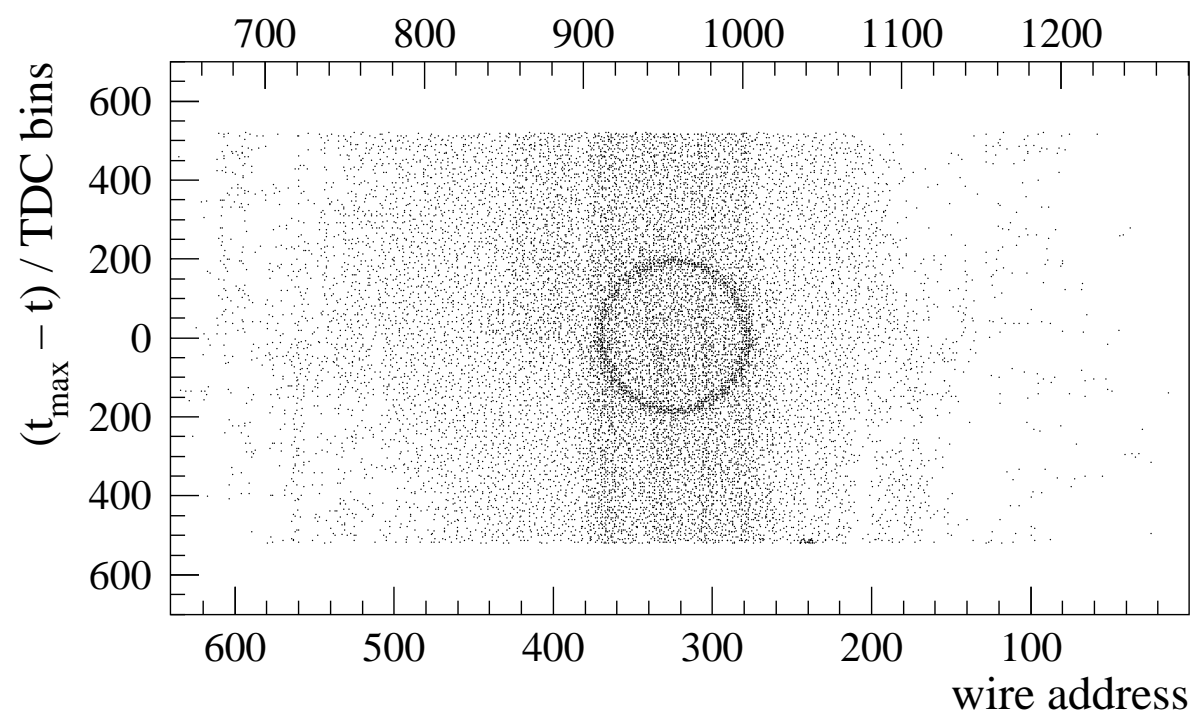

Figure 6: Timings vs wire address of signals from beam triggers. Horizontal axis: wire address; vertical axis: drift time $t_{\max }-t$ in TDC bins. 


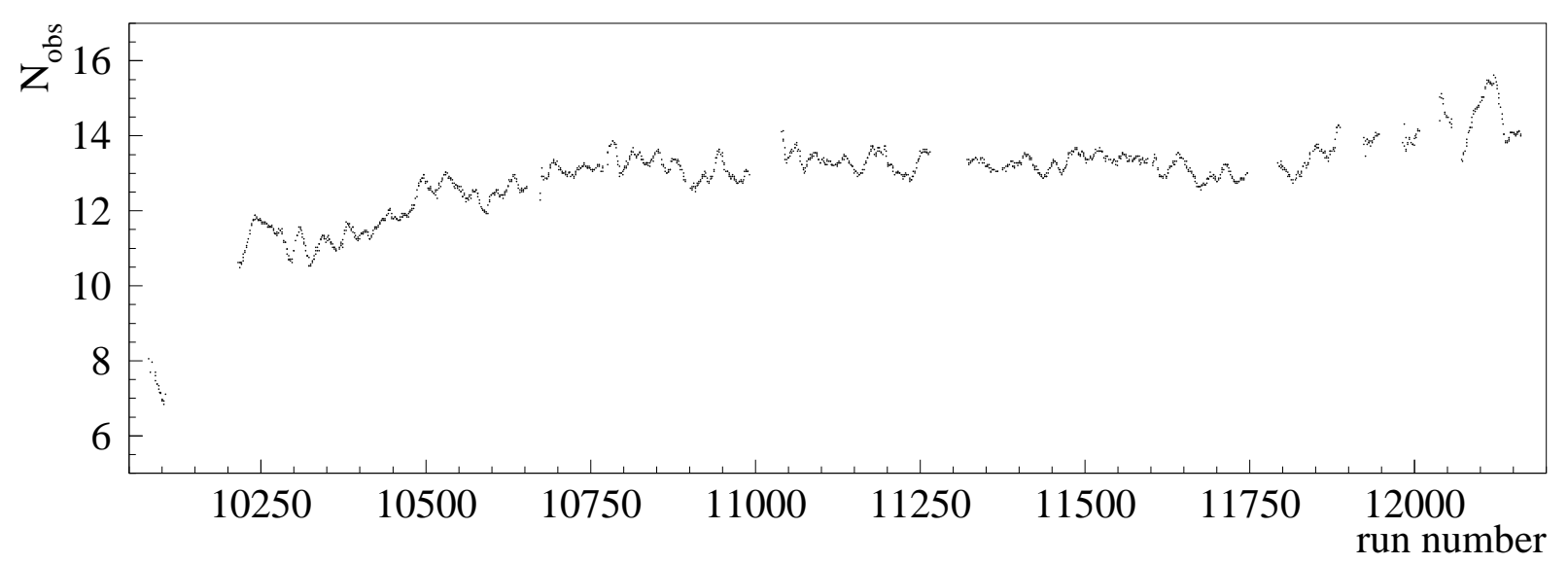

Figure 7: $N_{\text {obs }}$ vs run number in 1994 beam time. To obtain the number $N_{\text {pe }}$ of photoelectrons per ring, $N_{\text {obs }}$ has to be corrected for interactions and $\Sigma^{-}$decay losses: $N_{\text {obs }} \approx 0.70 \cdot N_{\mathrm{pe}}$.

From offline calibration of the 1993 beam time [15], the mean number of photoelectrons per ring, averaged over all chambers and all runs, was determined to be 15.5, corresponding to $N_{0}=55 \mathrm{~cm}^{-1}$.

\subsection{Background reduction}

The particle identification algorithm starts with the reduction of background hits. In addition to the high amplitude hits marked by the second discriminator threshold, clusters of at least six closely neighbouring hits are removed from the data. Furthermore, hits within regions of $1.5 \times 1.5 \mathrm{~cm}^{2}$ around the charged particle impact points are removed, as well as wires with 20 hits or more in an event. These parameters were optimized by two different criteria: the ratio of signal to the square root of background, and the efficiency and false identification probability for proton-pion separation [16]. Similar parameters were obtained with both criteria. Fig. 8 show the effect of background reduction on the distribution of ring radii. For the 1993 beam time, the number of background hits was reduced by $35 \%$ while $5.5 \%$ of the signal were lost.

\subsection{Particle identification}

A maximum likelihood approach is used for particle identification $[17,6]$. For each of the mass hypotheses $e, \pi, K$, and $p$ a probability density function for signal and background is computed at the position of every observed hit. The likelihood for each hypothesis is then calculated as the product of the function values of all hits, multiplied by an additional Poisson distribution for the total number of hits. To discriminate different particles, a cut on the ratio of the likelihoods is applied.

As an example for particle identification, Fig. 9 shows samples of $\overline{D^{0}} \rightarrow K^{+} \pi^{-}$and $D^{-} \rightarrow K^{+} \pi^{-} \pi^{-}$decays from 1993 data, with a mean $D$ momentum of $100 \mathrm{GeV} / \mathrm{c}$. For the solid curves, a weak RICH identification was already required during event selection, rejecting clearly identified pions and protons from the $K^{+}$candidates. Furthermore, the separation between interaction and decay vertex was required to be at least $3 \sigma$ of the experimental resolution.

For the hatched areas, $K^{+}$were selected using the likelihood ratio $r_{K}=\mathcal{L}_{K} /$ $\max \left(\mathcal{L}_{\pi}, \mathcal{L}_{p}\right)$, with the likelihoods defined as in ref. [17]. A cut on $r_{K}>1.3$ was applied for the $\overline{D^{0}}$, and $r_{K}>0.8$ for the $D^{-}$. In addition, the higher momentum $\pi^{-}$was 

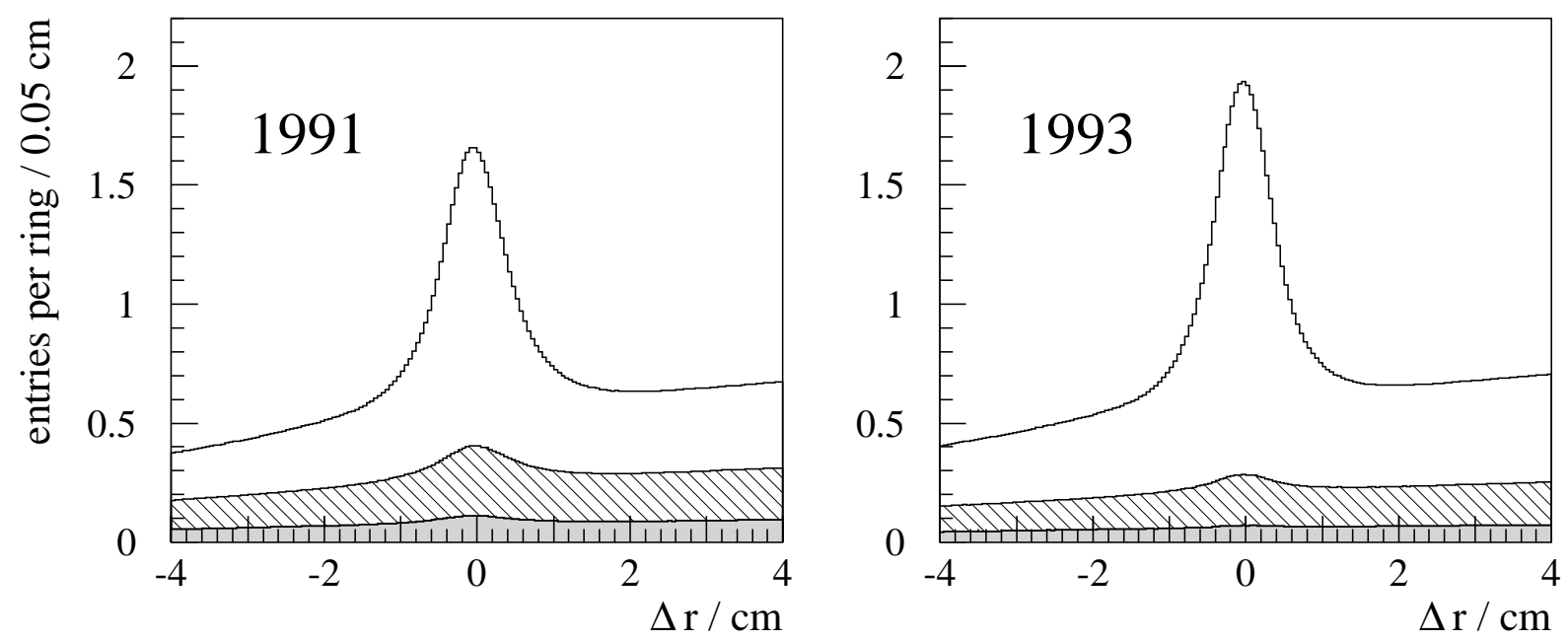

Figure 8: Effect of the two steps of background reduction for 1991 and 1993 data. The distance $\Delta r$ of hits from the predicted pion ring is plotted. Solid line: all hits; shaded area: high threshold hits; hatched area: software suppressed hits.
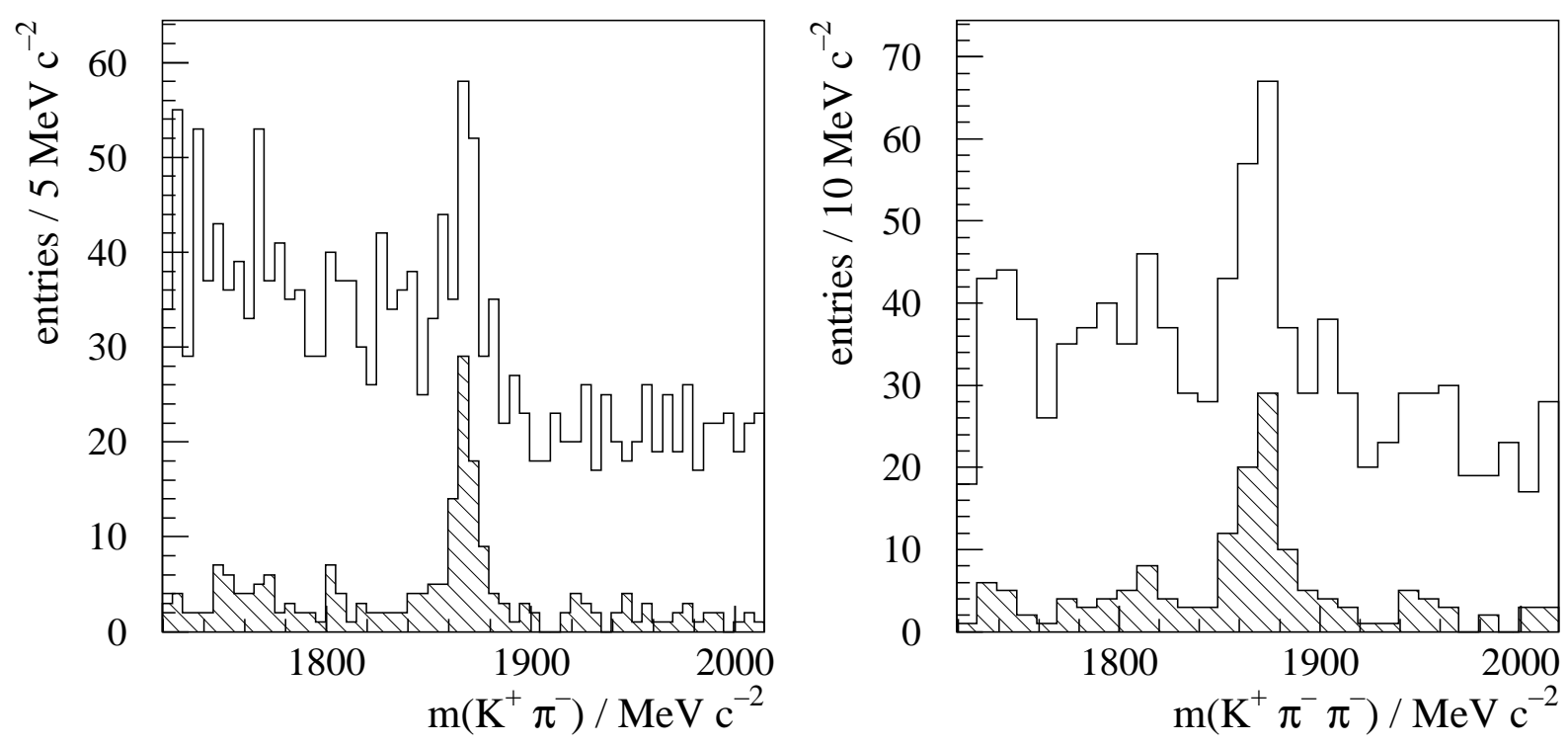

Figure 9: Invariant mass plots for $\overline{D^{0}} \rightarrow K^{+} \pi^{-}$(left) and $D^{-} \rightarrow K^{+} \pi^{-} \pi^{-}$(right), with weak (solid line) and strong (hatched area) RICH identification. 
identified in the case of the $D^{-}$. The background is reduced by about a factor of 15 , while almost all of the signal is kept.

\section{$5 \quad$ Conclusions}

The Omega RICH was reliably operated in the three physics beam times of WA89 in 1991, 1993, and 1994. It still requires a lot of careful maintenance and expertise, especially in the setup phase at the Omega "multi-user facility". In spite of this, its record of availability during data-taking is very good. During the run in 1991, its downtime was less than 5\%, and it had no downtime at all during the 1993 and 1994 runs.

A quality factor $N_{0}=55 \mathrm{~cm}^{-1}$ and a single photon resolution $\sigma_{\theta}=0.58 \mathrm{mrad}$ have been achieved. Providing $\pi / K$ separation up to momenta of $100 \mathrm{GeV} / \mathrm{c}$, the $\mathrm{RICH}$ is playing an essential rôle in the data analysis of the WA89 hyperon beam experiment.

\section{Acknowledgement}

We are indebted to J. Zimmer, A. Dallüge, and Y. Lesénéchal for their support during all phases of detector setup and operation.

\section{References}

[1] R. J. Apsimon et al., Nucl. Instr. Meth. A 248 (1986) 76; Nucl. Instr. Meth. A 241 (1985) 339.

[2] M. I. Adamovich et al., Phys. Lett. B 280 (1992) 163; Phys. Lett. B 305 (1993) 177.

[3] D. Elia et al., contributed paper to the RICH95 workshop, Uppsala, Sweden, June 1995; to be published in Nucl. Instr. Meth. A.

[4] M. I. Adamovich et al., Preprint CERN-PPE/95-105, submitted to Phys. Lett. B.

[5] K. Martens, doctoral thesis, Univ. Heidelberg (1994).

[6] U. Müller, doctoral thesis, MZ-KPH 23/94, Univ. Mainz (1994).

[7] M. I. Adamovich et al., Z. Phys. A 350 (1995) 379.

[8] A. Forino et al., CERN/SPSC/87-43, SPSC/P233 (1987); WA89 collaboration, CERN/SPSLC/93-2, SPSLC/M516 (1993).

[9] R. Abjean et al., C. R. Acad. Sci. (Paris) 271 B (1970) 411; A. Bideau-Méhu et al., Opt. Commun. 16 (1976) 186.

[10] A. Bideau-Méhu et al., J. Quant. Spectr. Rad. Transf. 25 (1981) 395; R. Abjean, A. Bideau-Méhu and Y. Guern, Nucl. Instr. Meth. A 354 (1995) 417.

[11] W. Beusch et al., Nucl. Instr. Meth. A 323 (1992) 373.

[12] B. Schmidt, Nucl. Inst. and Meth. A 252 (1986) 579; B. Schmidt and M. Roncossek, Austral. J. Phys. 45 (1992) 351.

[13] F. Hahn et al., Nucl. Instr. Meth. A 283 (1989) 686.

[14] P. Némethy et al., Nucl. Instr. Meth. A 212 (1983) 273; G. Wälder, diploma thesis, Univ. Heidelberg (1989).

[15] O. Thilmann, diploma thesis, Univ. Heidelberg (1994).

[16] M. Boss, diploma thesis, Univ. Heidelberg (1994).

[17] U. Müller et al., Nucl. Instr. Meth. A 343 (1994) 279. 